\title{
Determining some of the triggers for early life cycle failure in decay affected logistic queueing simulation
}
A. Bender ${ }^{1}$
A. Pincombe ${ }^{2}$
G. Sherman ${ }^{3}$

(Received 26 March 2010; revised 2 September 2010)

\begin{abstract}
Life-cycle cost estimates for large scale, long term, future military capabilities are difficult to make and subject to complexities. Usually they are generated from anecdotal experience. However, experience may not be a sound basis, so modelling and simulation are employed to define conditions that lead to early system failure in measures such as availability levels or the capability's life-of-type. Such models typically have common characteristics, including decay or degradation, queueing delays, availability of resources, and maintenance processes. Our generic model is a queue server, discrete event simulation that emulates macroscopic maintenance processes using time based parameters and statistical distributions. Previously we reported that the simulated system shows evidence of bifurcation-like behaviour in life-of-type estimates. This suggested that uncertainties in microscopic variables (such as inter-arrival times) cause instabilities in high level strategic perfor-
\end{abstract}

http://anziamj . austms.org.au/ojs/index.php/ANZIAMJ/article/view/2604 gives this article, (C) Austral. Mathematical Soc. 2010. Published October 1, 2010. ISSN 1446-8735. (Print two pages per sheet of paper.) Copies of this article must not be made otherwise available on the internet; instead link directly to this URL for this article. 
mance indicators, making the prediction of such indicators difficult and bringing into question the use of mean based estimation methods for inventory provisioning. Our objective is to define the conditions which lead to system failure. We use a series of numerical simulation experiments to investigate and define such conditions. Outcomes show that system performance is sensitive to the types of input distribution used and that decay processes are strongly associated with complex behaviour even when most of the interacting factors of the real system have been removed from the simulation.

\section{Contents}

1 Introduction

C716

2 The maintenance problem and modelling structure C718

3 Experimentation, results and analysis

4 Summary

C726

References

C728

\section{Introduction}

Complexities are likely to be involved in the maintenance and repair of large scale long term military vehicle systems, given the many factors which could interact to cause complex behaviour, whereas the existence of complexities in a model of such a system, after many of the interacting factors have been removed, is noteworthy. We previously showed that a model as simple as a single queue with identical servers and with several input distributions as well as a source of progressively increasing arrival rates, can exhibit complex 
behaviour such as bifurcations [1], with system lifetime varying by a factor of two or more over each set of Monte-Carlo runs. Early system failure was related to stochastic variations in the inputs - confirmed by removing the variations - and it seemed likely that the magnitudes of the variances could be important. Surprisingly, our trials showed that the variances of the input distributions had only a second order effect on the bifurcations. Thus we had evidence of complex behaviour, with no real pointers to the contributing factors. Other researchers $[2,5$, e.g.] reported results which suggest that simple queueing models can exhibit complex behaviour; however, there is no general understanding of the factors that are likely to contribute to complexity in such models. Our eventual aim is to determine the mechanisms which contribute to complex behaviour in our particular model. In this article we use a series of numerical experiments to identify those inputs which make some contribution to the occurrence of branching.

The repair cycle for a fleet of vehicles is represented as a single queue, multi-server arrangement with several input distributions, covering vehicle breakdowns, accidental damage, and scheduled maintenance. Performance is measured by the fraction of the vehicles available for use at any given time, and the parameters in the model are set so that, based on mean value estimation, the model performance should be satisfactory for greater than 300, 000 time steps. We use a Monte-Carlo approach, with multiple samples from each of the input distributions and we run multiple repeats for each set of parameter values. For non-zero variance in the input distributions, each set of repeats results in a mixture of successes and failures, where the system fails when vehicle availability falls below some threshold. Our approach is to systematically set the variances of particular distributions to zero and measure the occurrence of failures. Our results show that only two of the input distributions contribute to stochastically derived failure, with the major role being played by the distribution which includes a decay factor. 


\section{The maintenance problem and modelling structure}

Our model develops from a queueing theory perspective. The model is a discrete event simulation (DES) designed in the MATLAB/Simulink/SimEvent environment and emulates a G/D/c type of FIFO (First In First Out) queue and server system [1]. The various input profiles that determine inter-arrival and service times are discussed below.

The model was formulated to mimic the macroscopic behaviour of generic one-type vehicle fleet availability within a maintenance system. Figure 1 shows typical behaviour with respect to military vehicle fleet availability given as percentage of resources for some operational availability (OA) requirement with a life of type (LOT) around ten years. In this figure we assume an over allocation in resources of $10 \%$ above such a requirement that is represented by a sparing provision. Like many through life support systems it eventually undergoes a period of decline and decay. The curve in Figure 1 represents expected behaviour of a metamodel [4], such that the simulation model with known inputs should exhibit on average similar outcome behaviours.

The key outcome measure is availability (vehicles ready at any instant) where the model is based on a fleet of 600 vehicles, and the percentage availability is determined by the number of vehicles not in the maintenance queue server system.

The key features of our discrete event simulation model are

- Two different mechanisms for unscheduled failures are considered. The first one describes failures caused by material fatigue and ageing, and results in unscheduled maintenance requirements that increase slowly over time. Its functional form reflects experience with military (vehicle) capability systems, in which serviceability degrades gradually over the system lifetime. The second one generates a background of accidental, memoryless failures as caused, for instance, by vehicle accidents. 


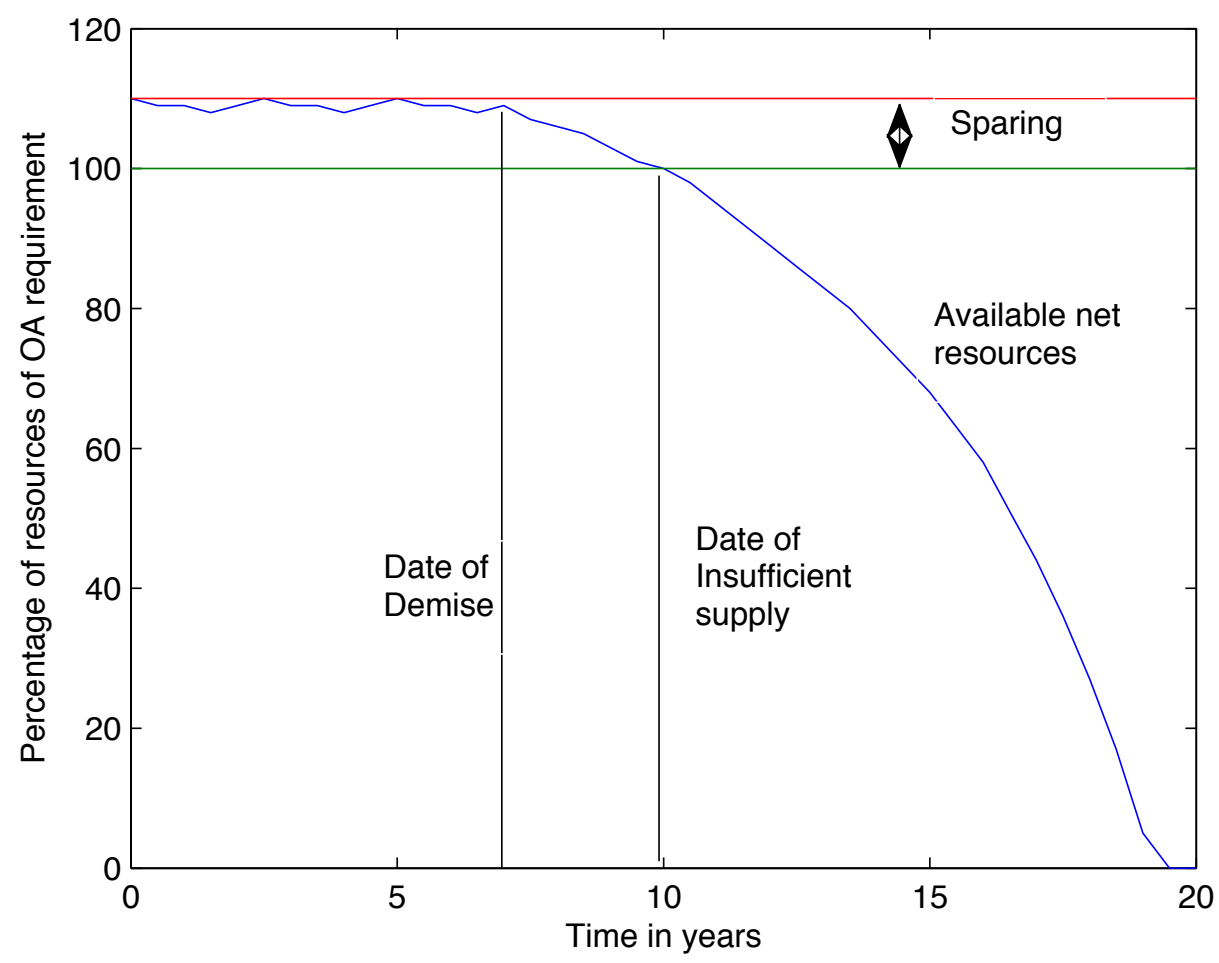

Figure 1: A typical life of type in available fleet stock levels. 
- Scheduled maintenance requirements are represented by a third distribution and this is overlaid with the two unscheduled inputs [5].

- Random variates describe the inherent uncertainties in failure rates and servicing times. They cause random, small time scale fluctuations in maintenance demand and server throughput. These fluctuations are very small in comparison to the total number of entities in the model; that is, they only perturb slightly a corresponding mean based inventory provisioning system.

- The number of servers is fixed (at 32 as in Experiment I [1]) and is not considered as part of the input profile.

- Average input inter-arrival times are designed as a function of time to incorporate a creeping decay. Arrival rates increase from approximately $88 \%$ to $99 \%$ of mean throughput capacity. This represents capacity planning based on a near maximum efficiency mean value model.

Five input profiles (random variates) are used in the simulation model of which two are found to be prominent in determining outcome characteristics. The first three represent statistical demand distributions $f(x)$ describing interarrival times for queue arrivals, with inter-arrival times then being used to mimic vehicle arrivals.

1. Decay random variate $(A=16, B=4, \lambda=-0.000005, \eta=0.1)$

$$
f(x ; t ; A ; B ; \lambda ; \eta)=\left(A+B e^{\lambda t}\right)\left\|1 / \mathcal{N}_{x}\left(1, \eta^{2}\right)\right\| .
$$

The gradual decay time is derived from a failure rate that satisfies the Verhulst equation giving a form of the logistic curve [6] and is often used in models for rates of systems failure in the process of aging [3]. The arrival rate for creeping decay is multiplied by a random variate Gaussian distribution with unity mean and a variable standard deviation which is determined in testing. This Gaussian distribution gives rise to small scale fluctuations that are present in real world repair demands caused by equipment degradation. 
2. Unscheduled requests (Exponential with $\beta=70$ )

$$
f(x ; \beta)= \begin{cases}\frac{1}{\beta} e^{-x / \beta}, & x \geqslant 0 \\ 0, & x<0 .\end{cases}
$$

3. Scheduled requests (Uniform with $a=60$ and $b=80$ )

$$
f(x)= \begin{cases}\frac{1}{b-a}, & \text { for } a \leqslant x \leqslant b \\ 0, & \text { for } x<a \text { or } x>b .\end{cases}
$$

The above two demand distributions describe regular and irregular maintenance arrival times. We use a randomised uniform distribution for inter-arrival times that relate to regular repair demands and a randomised exponential distribution (the distribution of times between events in a Poisson process) for those relating to irregular memoryless failure [5].

4. Service times (Gaussian) The repair server system is an $\mathrm{N}$-server $(\mathrm{N}=32)$. Repair processing times for each individual entity are sampled from a randomised Gaussian distribution (the fourth input) with mean $\mu=360$ and variance $\sigma^{2}=40$. When repairing the selected entity an entire server within the $\mathrm{N}$-server is busy for the whole randomly chosen time period.

5. Server downtime (negligible effect) Every 5000 time steps one server goes down for the same period of time it takes to service an entity (vehicle) - the net contribution though is less than $0.25 \%$ to throughput.

This system of inputs and model were tested in Experiment I [1]. The performance measures and outcomes of Experiment I then gave insight into why further experimentation is required in investigating possible causes of system failure. This experiment involved repetitive (Monte-Carlo) simulation for aggregating outcome statistics using the same parameter inputs/types of distributions with different randomisation seeds. Figure 2 explains the main 

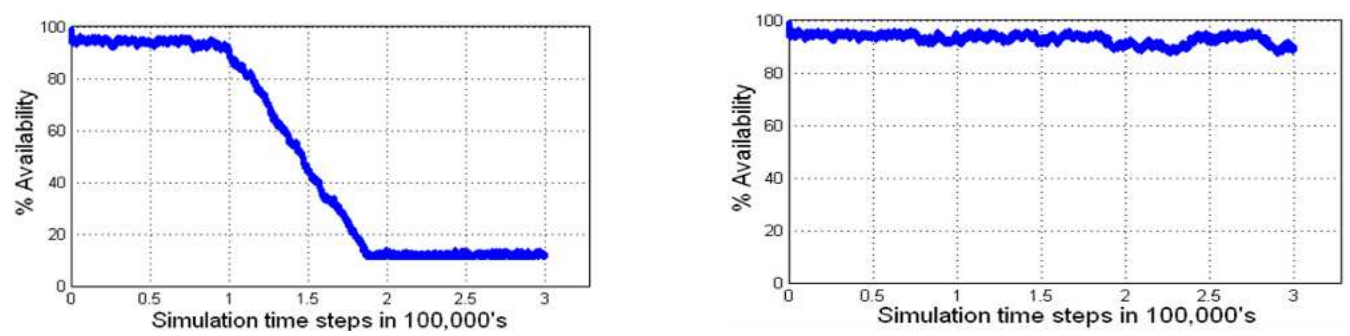

Figure 2: Typical output given by two DES model runs with the same input profiles (different randomisation seeds).

feature of Experiment I. Here the output given by two DES model runs with the same input profiles but with different randomisation seeds shows a failing LOT case (left) and a successful one (right).

The analysis of Experiment I indicated the following.

- Combined presence of fluctuations and decay gives rise to branching in availability outcomes, with consistent instability times.

- The creeping decay generates a particular type of branching in the loss of vehicles in maintenance.

- A possible explanation for this is that in some instances server queue behaviour for queue length (related to availability in our case) analytically behaves much like integral equations with memory effects of which some are known to have bifurcations. However, it seems solving queueing equations is too cumbersome and difficult to achieve an analytical expression in the case provided to verify this.

- Fluctuations in the service times of the servers do not seem to affect the branching process and cause only negligible changes in output compared to the branching effect. 


\section{Experimentation, results and analysis}

In earlier work [1] we found that different simulations using a sequence of differing random variates generated from identical input distributions could produce system lifetimes that varied by a factor of two or more, apparently via a branching or bifurcation process. The branching only occurred when the input variables (additions of vehicles to the repair system) had non-zero variances but, remarkably, the size of each variance otherwise contributed only second order effects to the results.

Three different input distributions are used in the model and we seek, in this article, to explore the contribution that each type of input makes to the branching (and therefore to the uncertainty in system lifetime). Input types make two contributions to the load on the repair system: they produce different numbers of vehicle arrivals, thus having different weights in their contributions to overall effects; and they involve stochastic variations which depend on the type and shape of the underlying distribution functions and associated parameters. One distribution type, covering vehicle breakdowns, also involves degradation of the equipment, so that the rate of arrivals increases over time. We use two processes to explore the occurrence of branching: first we vary the proportion of vehicle arrivals generated by each distribution; second, we run a series of numerical experiments, using a switch on the variance of each distribution to generate different combinations of stochastic and deterministic inputs. This method is known as screening and the approaches, screening by weighted contribution and $2^{k}$ factorial factor screening the fluctuations in the input profiles, are well accepted techniques.

In the first screening approach we look at the case of increasing and decreasing the contribution that the decay random variate has on the overall throughput of entities. We test the cases by adjusting the proportional contributions $\omega_{i}$ of the three arrival input distributions to the overall arrival rate, while the overall arrival rate is held constant $\left(\sum_{i} \omega_{i}=1\right)$. This was achieved by changing values $A, B, \beta, a$ and $b$ in equations (1), (2) and (3). 


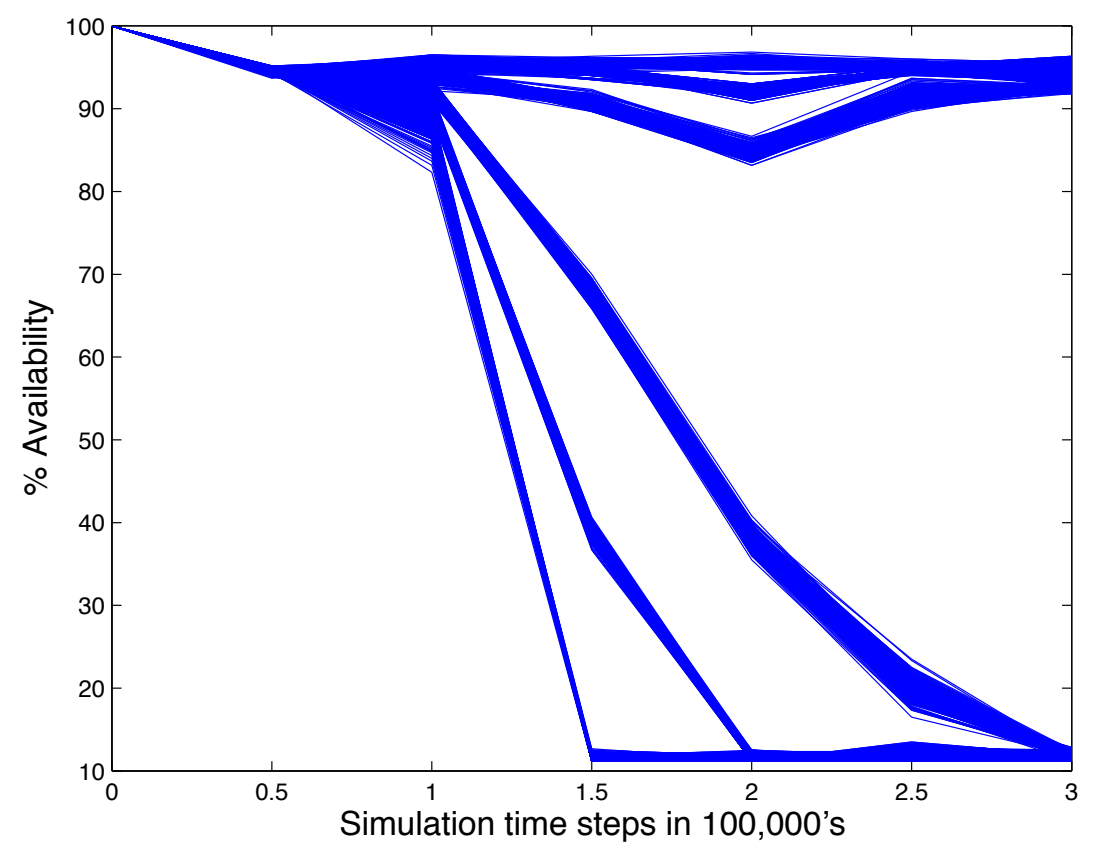

Figure 3: Availability levels when two-thirds of arrivals are caused by the decay random variate, one-sixth by unscheduled maintenance and one-sixth by scheduled maintenance. 


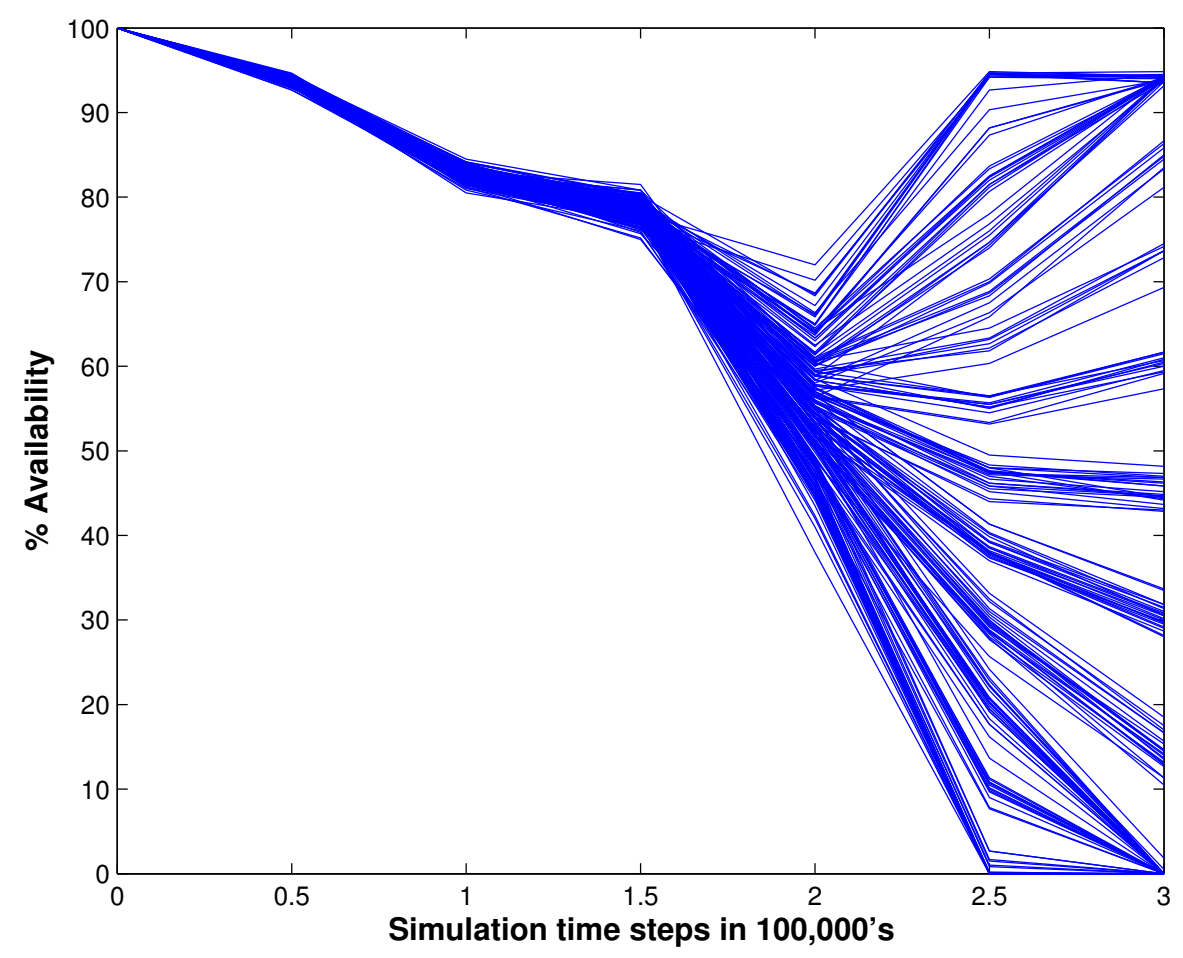

FIgURE 4: Availability levels when one-third of arrivals are caused by the decay random variate, one-third by unscheduled maintenance and one-third by scheduled maintenance. 
We test cases $\left(\omega_{1}, \omega_{2}, \omega_{3}\right)=(1,0,0),(2 / 3,1 / 6,1 / 6),(1 / 3,1 / 3,1 / 3)$ by running 300 simulations for each case. The initial case gives consistent early system failure and exhibits virtually no branching phenomenon. The second two are shown in Figure 3 and Figure 4, where each plot represents availability levels for the corresponding simulation runs sampled at every 50,000 time steps. Transfer of weight from the decay distribution results in a reduction in the tendency for early failure and a widening of the spread within each branch.

The second screening test investigates the output responses from selectively removing the fluctuations in each of the three arrival input profiles. When the randomisation is off, the decay input profile is set to $A+B e^{\lambda t}$, and the unscheduled and scheduled input profiles are set to a constant distribution of $\mu=70$. For each of the eight possible combinations of on/off fluctuation selection arrangements for these input profiles a Monte-Carlo experiment of 300 runs is performed. The observations from the eight Monte-Carlo tests are outlined in Table 1. The columns on the left hand side represent whether input profile 1 (IPr1 - the decay random variate), input profile 2 (IPr2 unscheduled) or input profile 3 (IPr3 - scheduled) has any variance at all in fluctuation.

Randomisation in the scheduled uniform input profile had a minimal effect on clustered sets of data about branching but did not affect branching overall. Removing fluctuation from the decay input profile has the largest effect on removing branching particularly when the Poisson variation is also turned off. The Poisson input causes some branching and does not cause system failure.

\section{Summary}

The presented study is motivated by model experimentation that incorporates decay processes and LOT issues into logistic DES models. There are aspects of such model behaviour which in many cases can be catastrophic to outcomes. We used factor screening techniques to determine possible causes of branching 
TABLE 1: A $2^{3}$ factorial test on switching on/off randomisation variance in the three arrival time distributions.

\begin{tabular}{|c|c|c|c|}
\hline IPr1 & IPr2 & IPr3 & Observation of output (on branching) \\
\hline on & on & on & $\begin{array}{l}\text { Substantial branching with some deviation } \\
\text { within branches }\end{array}$ \\
\hline on & on & off & $\begin{array}{l}\text { Substantial branching with some deviation } \\
\text { within branches }\end{array}$ \\
\hline on & off & on & $\begin{array}{l}\text { Substantial branching not much deviation } \\
\text { within branches }\end{array}$ \\
\hline on & off & off & $\begin{array}{l}\text { Substantial branching not much deviation } \\
\text { within branches }\end{array}$ \\
\hline off & on & on & Small branching triggered but not failing \\
\hline off & on & off & Small branching triggered but not failing \\
\hline off & off & on & Slight error around deterministic \\
\hline off & off & off & Deterministic \\
\hline
\end{tabular}

in output and system failure from the aspects of the input profiles. From our experimentation we observe that reducing the weighted contribution from decay causes the model to behave in a more predictive fashion. The evidence suggests that by reducing the contribution to input from decay the output exhibits less extreme branching outcomes and seems more like a fluctuating output measure bounded by envelope functions. The question then arises on whether the branching effects are a result of the input profile of the decay process or the inherent characteristic of fluctuation affecting a queueing process. The observations from factorial factor screening suggest the former, that the decay input profile is the prominent trigger for the branching process in the simulation output.

From an application perspective the better these behaviours are understood the more direction simulation and logistical planners have in designing suitable model structures. 


\section{References}

[1] Bender A., Pincombe A. and Sherman G. D., Effects of decay uncertainty in the prediction of life-cycle costing for large scale military capability projects 18th World IMACS / MODSIM Congress, Cairns, Australia 13-17 July 2009, http://mssanz.org.au/modsim09. C717, C718, C720, C721, C723

[2] Feichtinger G., Hommes C. H. and Herold W., Chaos in a Simple Deterministic Queueing System, ZOR - Mathematical Methods of Operations Research, 40, 1994, 109-119. doi:10.1007/BF01414032 C717

[3] Gavrilov L. A., Gavrilova, N. S., The reliability theory of ageing and longevity. Journal of Theoretical Biology, 213(4), 2001, 527-545. doi:10.1006/jtbi.2001.2430 C720

[4] Kleijnen, J. P. C., S. M. Sanchez, T. W. Lucas, and T. M. Cioppa, State-of-the-art review: a users guide to the brave new world of designing simulation experiments. INFORMS Journal on Computing, 17, no. 3, 2005, 263-289. doi:10.1287/ijoc.1050.0136 C718

[5] Upadhya S. K. and Srinivasan N. K., Availability of Weapon Systems with Logistic Delays: A Simulation Approach, International Journal of Quality 83 Reliability Management 20:7, 2004, 836-846. doi:10.1108/02656710310491249 C717, C720, C721

[6] Verhulst, P. F., Recherches mathematiques sur la loi d'accroissement de la population., Nouv. mem. de l'Academie Royale des Sci. et Belles-Lettres de Bruxelles 18, 1845, 1-41. C720

\section{Author addresses}

1. A. Bender, Land Operations Division, DSTO, Edinburgh, Australia. 
mailto:axel.bender@dsto.defence.gov.au

2. A. Pincombe, Land Operations Division, DSTO, Edinburgh, Australia.

3. G. Sherman, Land Operations Division, DSTO, Edinburgh, Australia. 